Comment. Math. Helv. 75 (2000) 171-188

(C) 2000 Birkhäuser Verlag, Basel

0010-2571/00/010171-18 \$1.50+0.20/0

Commentarii Mathematici Helvetici

\title{
Improper actions and higher connectivity at infinity
}

Kenneth S. Brown and John Meier*

\begin{abstract}
Given an improper action (= cell stabilizers are infinite) of a group $G$ on a CWcomplex $\mathcal{X}$, we present criteria, based on connectivity at infinity properties of the cell stabilizers under the action of $G$ that imply connectivity at infinity properties for $G$. A refinement of this idea yields information on the topology at infinity of Artin groups, and it gives significant progress on the question of which Artin groups are duality groups.
\end{abstract}

Mathematics Subject Classification (1991). 57M07, 20F65, $20 \mathrm{~F} 36$.

Keywords. Connectivity at infinity, Artin groups, duality groups.

\section{Introduction}

A locally finite, $m$-connected $\mathrm{CW}$ complex $\mathcal{X}$ is $m$-connected at infinity if, roughly speaking, $k$-spheres near infinity can be filled by $(k+1)$-balls near infinity for $-1 \leq k \leq m$. A group $G$ is said to be $m$-connected at infinity if $G$ acts freely and cocompactly on an $m$-connected complex $\mathcal{X}$ which is $m$-connected at infinity. This is a group theoretic property in that it is independent of the choice of locally finite space on which $G$ acts freely and with finite quotient. The condition (-1)connected at infinity is a fancy way of saying $G$ is infinite; 0 -connected at infinity is commonly referred to as "one-ended"; the group $\mathbb{Z}^{m}$ is $(m-2)$-connected at infinity. As Bestvina and Feighn point out [3], it is often possible to consider cocompact, proper (but not necessarily free) actions: A virtually torsion free group $G$ is $m$-connected at infinity if and only if it admits a cocompact, proper (= finite cell stabilizers) action on a locally finite, $m$-connected CW-complex which is $m$ connected at infinity. Also, important results about connectivity at infinity have been achieved starting with actions that are not cocompact: Bestvina and Feighn accomplish this for $\operatorname{Out}\left(F_{n}\right)$ [3]; Borel and Serre did the same for torsion free arithmetic groups [6]. This leaves open the question of what one can say about $G$ when $G$ acts improperly (= cell stabilizers are infinite) on a given complex $\mathcal{X}$.

${ }^{*}$ Meier thanks Cornell University for hosting him while on leave from Lafayette College, and the NSF for the support of an RUI grant, DMS 9705007. 
The only previous result along these lines is due to Jackson: If $G$ is the fundamental group of a finite graph of groups where the vertex stabilizers are 1connected at infinity and the edge stabilizers are one-ended, then $G$ is 1-connected at infinity [19]. Theorem A generalizes Jackson's result primarily in terms of moving beyond actions on trees to actions on cell complexes. Throughout this paper, a $G$-complex $\mathcal{X}$ is a combinatorial $\mathrm{CW}$ complex on which $G$ acts by permuting the cells. (For background on combinatorial complexes see [17].) We denote the isotropy group of a cell $\sigma \subset \mathcal{X}$ by $G_{\sigma}$. The action of $G$ on $\mathcal{X}$ is rigid if every induced action of an isotropy group $G_{\sigma}$ on its associated cell $\sigma$ is trivial.

Theorem A. Let $\mathcal{X}$ be a rigid $G$-complex with $G \backslash \mathcal{X}^{(m+1)}$ finite.

(i) If $\mathcal{X}^{(m+1)}$ is $m$-acyclic, and for each cell $\sigma \subset \mathcal{X}, G_{\sigma}$ is $F P_{m-|\sigma|+1}$ and $(m-|\sigma|)$-acyclic at infinity, then $G$ is $m$-acyclic at infinity.

(ii) Assume $\mathcal{X}^{(2)}$ is 1-connected, and: the vertex stabilizers are finitely presented and 1-connected at infinity; the edge stabilizers are finitely generated and one-ended; and the face stabilizers are infinite. Then $G$ is 1-connected at infinity.

(iii) If (in addition to the hypotheses of (i)) each vertex stabilizer $G_{v}$ is finitely presented and 1-connected at infinity, and $\mathcal{X}$ is 1-connected, then $G$ is $m$-connected at infinity.

(The topology at infinity terminology will be formally defined in the next section; we note that the condition $k$-acyclic at infinity for $k<-1$ is vacuous.)

We note that our conditions on $\mathcal{X}$ are very mild; it does not have to be locally finite, or have any connectivity at infinity properties. For example, $\mathcal{X}$ could be a tree where each vertex has countably infinite valence.

The "base case" of Theorem A states a known fact: If $G$ acts on a connected graph with compact quotient, where the vertex stabilizers are finitely generated and one-ended, and the edge stabilizers are infinite, then $G$ is one-ended. Because of the close connection between connectivity at infinity and duality properties of groups, we also get the following result.

Corollary. Let $G$ act on a contractible complex $\mathcal{X}$ with $G \backslash \mathcal{X}$ finite. If the cell stabilizers $G_{\sigma}$ are duality groups of dimension $d-|\sigma|$, then $G$ is a duality group of dimension $d$.

The most simple case of this Corollary occurs when $G$ decomposes as a free product with amalgamation, $G=A *_{C} B$. Here the Corollary states that if $A$ and $B$ are duality groups of dimension $d$, and $C$ is a duality group of dimension $(d-1)$, then $G$ is a duality group of dimension $d$. This result was noted by Bieri, and his proof uses a Mayer-Vietoris sequence [4]. It seems appropriate that the proof of part (i) of Theorem A uses a spectral sequence argument, and this spectral sequence immediately establishes the Corollary. The proof of part (ii) is very geometric and borrows tools from the theory of complexes of groups. For 
those familiar with the terminology, our results could be stated in those terms. For example, part (ii) of Theorem A could be stated as: Let $G$ be the fundamental group of a finite, developable 2-complex of groups where: the vertex groups are finitely presented and 1-connected at infinity; the edge groups are finitely generated and one-ended; and the face groups are infinite. Then $G$ is 1-connected at infinity.

Because there is a pro-Hurewicz Theorem, stating that a complex which is $m$ acyclic at infinity and 1-connected at infinity is $m$-connected at infinity (see [20] or [15]), parts (i) and (ii) imply part (iii) of Theorem A.

In the last sections we outline how one can use these techniques to discuss connectivity at infinity properties of Artin groups. Given a finite simplicial graph $\mathcal{G}$, with edges labelled by integers greater than one, the associated Artin group, denoted $A_{\mathcal{G}}$, has a finite presentation with generators corresponding to the vertices of $\mathcal{G}$, and relations

$$
\underbrace{a b a \cdots}_{n \text { letters }}=\underbrace{b a b \cdots}_{n \text { letters }}
$$

where $\{a, b\}$ is an edge of $\mathcal{G}$ labelled $n$. (References include [1], [10], [14] and [25].)

Given any Artin group $A_{\mathcal{G}}$ there is an associated Coxeter group $C_{\mathcal{G}}$ which is the quotient of $A_{\mathcal{G}}$ formed by adding the relations $v^{2}=1$ for each generator $v$. An Artin group is of finite type if its associated Coxeter group is finite. Let $\widehat{\mathcal{G}}$ be the simplicial complex formed by attaching a simplex $\sigma$ of appropriate dimension to each complete subgraph $\mathcal{C} \subset \mathcal{G}$ for which $A_{\mathcal{C}}$ is an Artin group of finite type.

Charney and Davis describe a "modified Deligne complex" $\Phi_{\mathcal{G}}$ on which $A_{\mathcal{G}}$ acts with the cone of $\widehat{\mathcal{G}}$ as fundamental domain [10]. The action is improper; cell stabilizers are Artin groups of finite type. By modifying the arguments given for Theorem A, and applying them to this action, we establish the following two results.

Theorem B. Let $A_{\mathcal{G}}$ be an Artin group, let $\widehat{\mathcal{G}}$ be the complex described above, and assume that $\mathcal{G}$ is not a single vertex or edge. If $\widehat{\mathcal{G}}$ is 1 -connected and contains no cut vertex, then $A_{\mathcal{G}}$ is 1-connected at infinity.

Theorem C. Let $A_{\mathcal{G}}$ be an Artin group and assume $\Phi_{\mathcal{G}}$ is contractible. If $\widehat{\mathcal{G}}$ is Cohen-Macaulay, then $A_{\mathcal{G}}$ is a duality group.

Charney and Davis give general conditions implying that $\Phi_{\mathcal{G}}$ is contractible, and they conjecture that $\Phi_{\mathcal{G}}$ is always contractible (Conjecture 2 of [10]). We also note that these results partially extend work in [7] which completely determines connectivity at infinity and duality properties for right-angled Artin groups; there is also a strong parallel with Davis's results on duality for Coxeter groups [12]. Theorem $\mathrm{C}$ gives substantial progress toward resolving a question of Mike Davis about which Artin groups are duality groups (see Question 2 in [7]). 


\section{Definitions \& background}

A group $G$ is $\mathcal{F}_{m}$ if there is a $K(G, 1)$ with finite $m$-skeleton; $G$ is $F P_{m}$ if there is a projective resolution of $\mathbb{Z}$ as a trivial $\mathbb{Z} G$-module that's finite through dimension $m$. The action of $G$ on the universal cover of a $K(G, 1)$ provides a free resolution of $\mathbb{Z}$, so $\mathcal{F}_{m} \Rightarrow F P_{m}$, but the converse is false for $m>1$ [2]. A group is of type $F P$ if there is a finite projective resolution of $\mathbb{Z}$ as a trivial $\mathbb{Z} G$-module.

Let $G$ be $\mathcal{F}_{m+1}$ and let $\mathcal{Y}$ be the $(m+1)$-skeleton of the universal cover of a $K(G, 1)$. The group $G$ is $m$-connected at infinity if given any compact set $C \subset \mathcal{Y}$ there is a compact subcomplex $D \subset \mathcal{Y}$ such that any map $\phi: S^{k} \rightarrow \mathcal{Y}-D$ extends to a map $\hat{\phi}: B^{k+1} \rightarrow \mathcal{Y}-C$ for $-1 \leq k \leq m$. We call $D$ the $m$-connectivity subcomplex associated to $C$. We note that our condition "1-connected at infinity" is stronger than what some authors term "simply connected at infinity"; although the literature is not consistent on terminology, some would consider finitely generated free groups to be simply connected at infinity.

If $G$ is of type $F P_{m+1}$ and $H^{k}(G, \mathbb{Z} G)=0$ for $-1 \leq k \leq m+1$ and $H^{m+2}(G, \mathbb{Z} G)$ is $\mathbb{Z}$-torsion free, then $G$ is $m$-acyclic at infinity. This definition can be made more geometrically intuitive in the presence of stronger finiteness conditions. When $G$ is $\mathcal{F}_{m+1}$, one can say that $G$ is $m$-acyclic at infinity if any $k$-cycle supported in $\mathcal{Y}-D$ is the boundary of a $(k+1)$-chain supported in $\mathcal{Y}-C$. In particular, one has the following result (see $\S 4.3$ and $\S 5.5$ in [15]).

Proposition 2.1. Let $\mathcal{X}$ be a $K(G, 1)$ complex with $\mathcal{X}^{(m+1)}$ finite, and let $\left\{K_{i}\right\}$ be a nested, exhaustive sequence of compact subcomplexes of $\tilde{\mathcal{X}}^{(m+1)}$. Then the following are equivalent.

(i) $G$ is $m$-acyclic at infinity;

(ii) The sequences of reduced homology groups $\widetilde{H}_{k}\left(\widetilde{\mathcal{X}}^{(m+1)}-K_{i} ; \mathbb{Z}\right)$ are protrivial for $-1 \leq k \leq m$; and

(iii) $H^{k}(G, \mathbb{Z} G)=0$ for $-1 \leq k \leq m+1$ and $H^{m+2}(G, \mathbb{Z} G)$ is $\mathbb{Z}$-torsion free.

According to our definitions, in order for a group to be $m$-acyclic at infinity, it must be $F P_{m+1}$. Hence it is natural that in Theorem A we have finiteness conditions along with connectivity at infinity conditions on our isotropy groups. But in order for Theorem A to even make sense we would need $G$ to be $F P_{m+1}$ or $\mathcal{F}_{m+1}$, which we did not explicitly require. However, this follows from our hypotheses by the following result.

Theorem 2.2. ([9]) If $G$ acts on an m-acyclic complex $\mathcal{X}$ with $G \backslash \mathcal{X}^{(m+1)}$ finite, and for each cell $\sigma \subset \mathcal{X}, G_{\sigma}$ is $F P_{m-|\sigma|}$, then $G$ is $F P_{m}$. Further, if the vertex stabilizers are finitely presented, and $\mathcal{X}$ is simply connected, then $G$ is $\mathcal{F}_{m}$.

In our proof of Theorem A we use the technology of complexes of groups. In particular, we focus on the following two-dimensional case. Say $\mathcal{X}$ is a 1-connected, 
rigid $G$-2-complex with $G \backslash \mathcal{X}$ finite. Then there is a free $G$-2-complex $\mathcal{Y}$ and a $G$ equivariant cellular projection $\pi: \mathcal{Y} \rightarrow \mathcal{X}$ which we'll describe below. Because of our conditions, $G \backslash \mathcal{Y}$ is finite, so to establish part (ii) of Theorem A it suffices to show that $\mathcal{Y}$ is 1 -connected at infinity.

The space $\mathcal{Y}$ and the projection $\pi: \mathcal{Y} \rightarrow \mathcal{X}$ have a number of useful properties:

(i) The fibres over vertices, $\pi^{-1}(v)$, are Cayley complexes for the isotropy groups $G_{v}$.

(ii) The subspace $\pi^{-1}(e), e$ being an open edge, has a product structure. The fibre over the barycenter $b_{e}$ of $e$ is a Cayley graph for $G_{e}$, and $\pi^{-1}(e) \simeq$ $\pi^{-1}\left(b_{e}\right) \times(0,1)$. The closure of $\pi^{-1}(e), \overline{\pi^{-1}(e)}$, looks like a product $\pi^{-1}\left(b_{e}\right) \times$ $[0,1]$ where $\pi^{-1}\left(b_{e}\right) \times\{0\}$ is a copy of the Cayley graph $\pi^{-1}\left(b_{e}\right)$ inside of the Cayley complex $\pi^{-1}(\iota(e))$; such a copy exists because $G_{e}$ injects into $G_{\iota(e)}$. Similarly, $\pi^{-1}\left(b_{e}\right) \times\{1\}$ is a copy of $\pi^{-1}\left(b_{e}\right)$ in $\pi^{-1}(\tau(e))$.

(iii) For any open 2-cell $f \subset \mathcal{X}, \pi^{-1}(f)$ is $G_{f} \times B$ where $B$ is an open 2cell. Given an element $g_{f} \in G_{f}, g_{f} \times B$ is attached to an edge path circuit as follows. Let $f$ be an $n$-gon, with bounding edges $\left\{e_{1}, \ldots, e_{n}\right\}$, where $v_{i}=\iota\left(e_{i}\right)$, and let $\partial \bar{B}$ be divided into $2 n$ pieces labelled $\left\{\widetilde{v}_{1}, \widetilde{e}_{1}, \ldots, \widetilde{v}_{n}, \widetilde{e}_{n}\right\}$. Each $\widetilde{e}_{i}$ attaches to the edge in $\pi^{-1}\left(e_{i}\right)$ corresponding to the image of $g_{f}$ in $G_{e_{i}}$ induced by $G_{f} \hookrightarrow G_{e_{i}}$; the edges $\widetilde{v}_{i}$ are sent to paths in $\pi^{-1}\left(G_{v_{i}}\right)$ connecting the end vertex of $\widetilde{e}_{i-1}$ to the initial vertex of $\widetilde{e}_{i}$.

The construction of the complex $\mathcal{Y}$ and projection $\pi$ is very similar to the Scott and Wall "ball-and-stick" construction for groups acting on simplicial trees [24]. For details on the construction in dimension two, see [11], [16] and [23]. Note: This construction can be carried out in any dimension (see [16]), however we only require it in dimension two.

In [5], Bieri and Eckmann introduce the idea of a duality group, generalizing Poincaré duality groups. An FP group $G$ is a duality group if there is an integer $n$ and a $G$-module $D$ (the dualizing module) such that $H^{i}(G, M)$ $\approx H_{n-i}(G, D \otimes M)$ for all integers $i$ and $G$-modules $M$. Equivalently, it's a duality group if its cohomology with group ring coefficients is torsion free and concentrated in dimension $n$; in this case the dualizing module $D$ is $H^{n}(G, \mathbb{Z} G)$. So once one knows that an FP group $G$ has cohomological dimension $n$, one only needs to establish that $G$ is $(n-2)$-acyclic at infinity in order to show that $G$ is a duality group. (See [4], [8], and [13] for further information.)

\section{Homology at infinity}

In establishing part (i) of Theorem A, we use the following lemma, which may be well known. While cohomology commutes with direct sums in the presence of strong finiteness conditions - such as the FP condition - Theorem A (i) only assumes $G$ has more limited finiteness properties, such as the $F P_{m}$ property. 
Lemma 3.1. Let $G$ be a group of type $F P_{m}$ and let $\left\{M_{i}\right\}$ be a collection of G-modules.

(i) The canonical map $\bigoplus_{i} H^{m}\left(G, M_{i}\right) \rightarrow H^{m}\left(G, \bigoplus_{i} M_{i}\right)$ is an isomorphism. In particular, if $H^{m}\left(G, M_{i}\right)=0$ for all $i$, then $H^{m}\left(G, \bigoplus_{i} M_{i}\right)=0$.

(ii) If $M_{i}$ and $H^{m+1}\left(G, M_{i}\right)$ are $\mathbb{Z}$-torsion free for all $i$, then $H^{m+1}\left(G, \bigoplus_{i} M_{i}\right)$ is $\mathbb{Z}$-torsion free.

Proof. (i) By hypothesis there is a partial free resolution

$$
F_{m} \rightarrow F_{m-1} \rightarrow \cdots \rightarrow F_{1} \rightarrow F_{0} \rightarrow \mathbb{Z} \rightarrow 0
$$

where each $F_{i}$ is a finitely generated free $\mathbb{Z} G$-module. Let $K=\operatorname{im}\left\{F_{m} \rightarrow F_{m-1}\right\}$. Then an $m$-cocycle of $G$ with coefficients in a $G$-module $M$ can be identified with a $G$-module homomorphism $u: K \rightarrow M$, and $u$ is a coboundary if and only if $u$ extends to a map $F_{m-1} \rightarrow M$ (see the proof of Lemma VIII.2.1 in [8]). In other words, $H^{m}(G, M)$ is the cokernel of the restriction map

$$
\operatorname{Hom}_{G}\left(F_{m-1}, M\right) \rightarrow \operatorname{Hom}_{G}(K, M) .
$$

Since $F_{m-1}$ and $K$ are finitely generated, it follows that $H^{m}(G,-)$ preserves direct sums, whence (i).

(ii) Let $L=\operatorname{ker}\left\{F_{m} \rightarrow F_{m-1}\right\}$. As above, $H^{m+1}(G, M)$ is the cokernel of

$$
\operatorname{Hom}_{G}\left(F_{m}, M\right) \rightarrow \operatorname{Hom}_{G}(L, M) .
$$

Let $u: L \rightarrow \bigoplus_{i} M_{i}$ represent a torsion element of $H^{m+1}\left(G, \bigoplus_{i} M_{i}\right)$. Then some multiple $k \cdot u(k \neq 0)$ extends to a map $F_{m} \rightarrow \bigoplus_{i} M_{i}$. Since $F_{m}$ is finitely generated, all but finitely many components of this map are trivial, so the same is true of $k \cdot u$ and hence of $u$. (This last assertion uses our assumption that $M_{i}$ is $\mathbb{Z}$-torsion free.)

There is then a family of cocycles $u_{i}: L \rightarrow M_{i}$ such that almost all are trivial and all represent torsion elements of $H^{m+1}\left(G, M_{i}\right)$. Since the latter is torsion free, we may extend $u_{i}$ to $\tilde{u}_{i}: F_{m} \rightarrow M_{i}$, taking $\tilde{u}_{i}=0$ for almost all $i$. These $\tilde{u}_{i}$ 's give an extension $\tilde{u}: F_{m} \rightarrow \bigoplus_{i} M_{i}$ of $u$. Thus $u$ represents the trivial element of $H^{m+1}\left(G, \bigoplus_{i} M_{i}\right)$.

Proof of part (i) of Theorem A. By Proposition 2.1, we need to establish that $H^{i}(G, \mathbb{Z} G)=0$ for $0 \leq i \leq m+1$ and $H^{m+2}(G, \mathbb{Z} G)$ is $\mathbb{Z}$-torsion free. We use 
the well-known spectral sequence

$$
E_{1}^{p q}=\prod_{\sigma \in \Sigma_{p}} H^{q}\left(G_{\sigma}, \mathbb{Z} G\right) \Rightarrow H^{p+q}(G, \mathbb{Z} G)
$$

where $\Sigma_{p}$ is a set of representatives for the $G$-orbits of $p$-cells. (See for instance $\S$ VII.7 of [8].) If $\sigma \in \Sigma_{p}$ our hypothesis says that $G_{\sigma}$ is $F P_{m-p+1}$ and that $H^{q}\left(G_{\sigma}, \mathbb{Z} G_{\sigma}\right)$ vanishes for $q \leq m-p+1$ and is $\mathbb{Z}$-torsion free for $q=m-p+2$. Now $\mathbb{Z} G$, as a $G_{\sigma}$-module, is a direct sum of copies of $\mathbb{Z} G_{\sigma}$; so Lemma 3.1 implies that $E_{1}^{p q}$ vanishes for $p+q \leq m+1$ and is $\mathbb{Z}$-torsion free for $p+q=m+2$. The same is therefore true of $E_{\infty}^{p q}$, so $H^{i}(G, \mathbb{Z} G)$ vanishes for $i \leq m+1$ and is $\mathbb{Z}$-torsion free for $i=m+2$.

We note that in the proof of this homological version of Theorem $\mathrm{A}$, the only place we used the fact that the action of $G$ on $\mathcal{X}^{(m+1)}$ is cocompact is in establishing finiteness properties of $G$; if one already knows that $G$ is $F P_{m+1}$ then the hypothesis that the quotient is finite can be dropped.

\section{Simple connectivity at infinity}

Let $\mathcal{X}$ be a 1 -connected $G$-2-complex, where $\mathcal{X}$ and the $G$-action satisfy the conditions of part (ii) of Theorem A. Let $\mathcal{Y}$ be the free $G$-2-complex and let $\pi: \mathcal{Y} \rightarrow \mathcal{X}$ be the $G$-equivariant surjection (briefly) described in $\S 2$. The space $\mathcal{Y}$ is 1 -connected with $G \backslash \mathcal{Y}$ finite, so our goal is to show that $\mathcal{Y}$ is 1 -connected at infinity. That it is one-ended follows from part (i) of Theorem A.

Start with a finite subcomplex $C \subset \mathcal{Y}$. Recall that for each $v \in \mathcal{X}^{(0)}$, $\pi^{-1}(v)$ is 1-connected at infinity. Let $C_{v}=C \cap \pi^{-1}(v)$. If $C_{v}$ is empty, set $D_{v}=\emptyset$. Otherwise let $D_{v}$ be the 1-connectivity at infinity subcomplex in $\pi^{-1}(v)$ that is associated with $C_{v}$. For convenience we will assume $D_{v}$ is sufficiently large so that $\pi^{-1}(v)-D_{v}$ is connected. Set $C^{\prime}=C \cup\left(\bigcup_{v \in \mathcal{X}^{(0)}} D_{v}\right)$.

For any edge $e \subset \mathcal{X}, \overline{\pi^{-1}(e)}$ is the product of a Cayley graph for $G_{e}$ with $[0,1]$. Let $C_{e}^{\prime}$ be $C^{\prime} \cap \overline{\pi^{-1}(e)}$ and set $D_{e}^{\prime}$ to be the empty set when $C_{e}^{\prime}$ is empty. If $C_{e}^{\prime}$ is not empty, then the projection from $\overline{\pi^{-1}(e)}$ onto $\pi^{-1}\left(b_{e}\right)$ describes a subgraph $\widehat{C}_{e}$ of the Cayley graph $\pi^{-1}\left(b_{e}\right)$. Let $\widehat{D}_{e}$ be the 0 -connectivity at infinity subgraph of $\pi^{-1}\left(b_{e}\right)$ corresponding to $\widehat{C}_{e}$, and let $D_{e}^{\prime}$ be the union of 2-cells in $\overline{\pi^{-1}(e)}$ given

by the product of $\widehat{D}_{e}$ with $[0,1]$. Finally, set $D=C^{\prime} \cup\left(\bigcup_{e \in \mathcal{X}^{(1)}} D_{e}^{\prime}\right)$. We will show that any edge path circuit $p \subset \mathcal{Y}-D$ is null-homotopic in $\mathcal{Y}-C$. It suffices to consider simple edge path circuits, and we restrict ourselves to that case. We note that if the image of $p$ in $\mathcal{X}, \pi(p)$, is a single vertex $v$ then $p$ is contained in $\pi^{-1}(v)-D_{v}$, and hence it is null-homotopic in $\pi^{-1}(v)-C_{v}$ by our choice of $D_{v}$. 


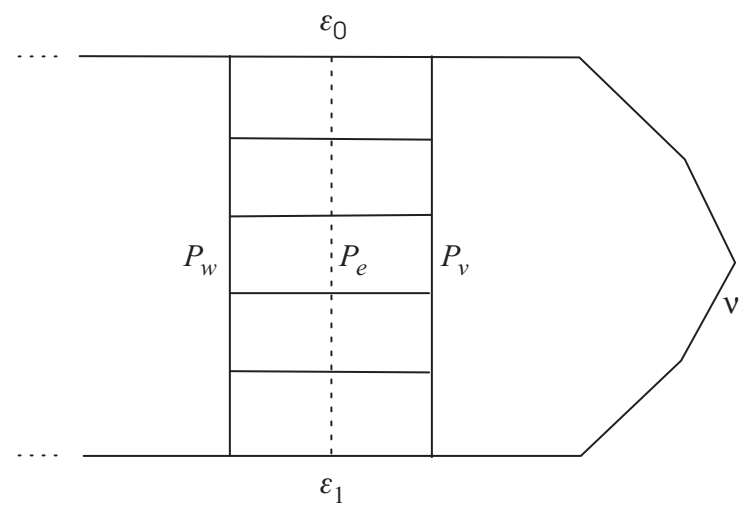

Figure 1. Joining the edges $\epsilon_{0}$ and $\epsilon_{1}$.

In the arguments which follow we will be subdividing the path $p$ according to which $\pi$-preimages it runs through. A local subpath of $p$ is a maximal subpath of $p$ that is contained in the $\pi$-preimage of a single vertex in $\mathcal{X}$. The path $p$ can be viewed as the union of its local subpaths, where each pair of consecutive local subpaths are joined by a single edge contained in the $\pi$-preimage of an edge in $\mathcal{X}$.

Lemma 4.1. It suffices to consider only those simple edge path circuits $p \subset \mathcal{Y}-D$ where $\pi(p) \subset \mathcal{X}$ is also a simple edge path circuit.

Proof. Assume first that some subpath $p^{\prime}$ of $p$ projects onto a simple circuit in $\mathcal{X}$ that is a proper subset of the entire image. Let the vertices of this subpath $p^{\prime}$ be $\{a, b, \ldots, z\}$. Since the image of $p^{\prime}$ is a simple circuit, $\pi(a)=\pi(z)=v$. But $\pi^{-1}(v)$ is one-ended, hence by our choice of the subcomplex $D_{v}$, there is a path $p^{\prime \prime}$ in $\pi^{-1}(v)-D_{v}$ connecting a to $z$. Thus $p^{\prime} \cup p^{\prime \prime}$ is a simple circuit in $\mathcal{Y}-D$ projecting onto a simple circuit in $\mathcal{X}$, and $\left(p-p^{\prime}\right) \cup p^{\prime \prime}$ is a simple circuit in $\mathcal{Y}-D$ projecting onto a graph in $\mathcal{X}$ containing one fewer simple sub-circuit.

This process reduces us to the case where $\pi(p)$ is a simple circuit, or a tree. It remains then to resolve the possibility that $\pi(p)$ is a tree. Let $v \in \pi(p)$ be a vertex of valence 1 in $\pi(p)$, let " $e$ " be the edge in $\pi(p)$ which is attached to $v$, and let $w$ be the other vertex of $e$. Then in $p$ there is a subpath $p^{\prime}$ consisting of an edge $\varepsilon_{0}$ followed by a local subpath $\nu$, and concluding with an edge $\varepsilon_{1}$, where $\pi\left(\varepsilon_{i}\right)=e$ and $\pi(\nu)=v$. The barycenters of the edges $\varepsilon_{i}$ correspond to vertices in the Cayley graph of $G_{e}$ that are outside of $\widehat{D}_{e}$. By our choice of $\widehat{D}_{e}$, there is a path $p_{e}$ connecting $b_{\varepsilon_{0}}$ to $b_{\varepsilon_{1}}$ in $\pi^{-1}\left(b_{e}\right)-\widehat{D}_{e}$. The induced collection of 2-cells in $\overline{\pi^{-1}(e)}$ connects the edges $\varepsilon_{0}$ and $\varepsilon_{1}$ in $\mathcal{Y}-D$. The path $p_{e}$ (in the Cayley graph of $G_{e}$ ) projects to paths $p_{v}$ and $p_{w}$ contained in the $\pi$-preimages of $v$ and $w$ respectively. Combining $p_{v}$ with $\nu$ gives a circuit in $\pi^{-1}(v)-D_{v}$ that by hypothesis can be filled outside of $\pi^{-1}(v)-C_{v}$. Using this filling, along with the 2-cells in $\pi^{-1}(e)$ 
induced by $p_{e}$, allows us to replace the subpath $p^{\prime}\left(=\varepsilon_{0} \cup \nu \cup \varepsilon_{1}\right)$ by the path $p_{w}$. Thus it remains to find a filling for an edge path circuit whose image in $\mathcal{X}$ is a tree with one less edge - the edge connecting $w$ to $v$ having been removed — and this we can do by induction.

Proof of Theorem A (ii). Our proof is highly geometric, so we strongly recommend that the reader sketch several pictures of their own as they work through this proof.

Because $\mathcal{X}$ is 1-connected, there is a cellular 2-disk $K$ and a combinatorial map $\phi:(K, \partial K) \rightarrow(\mathcal{X}, \pi(p))$. We use $K$ as a guide to constructing a cellular 2-disk $\widetilde{K}$ and a map $\widetilde{\phi}:(\widetilde{K}, \partial \widetilde{K}) \rightarrow(\mathcal{Y}, p)$, where $\widetilde{\phi}(\widetilde{K}) \subset \mathcal{Y}-C$. In particular, we construct a cellular projection $\varpi: \widetilde{K} \rightarrow K$ such that the following diagram commutes:

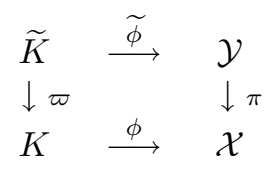

The disk $\widetilde{K}$ is essentially a complex formed by expanding all the vertices and edges of $K$ to polygons, and then attaching these polygons to each other in a manner prescribed by the intersections in $K$. We describe this "blowing up" process in detail below.

Islands: For each $n$-gon $f \subset K$, let $I_{f}$ be a $(2 n)$-gon, which we will refer to as an island. Because $\phi: K \rightarrow \mathcal{X}$ is combinatorial, we think of $f$ as a cell in $\mathcal{X}$. So $\partial f$ can be thought of as an edge path determined by a sequence of vertices $\left\{v_{1}, v_{2}, \ldots v_{n}\right\} \subset \mathcal{X}^{(0)}$; let $e_{i}$ be the edge $\left[v_{i}, v_{i+1}\right]$ (with indices taken modulo $n$ ). Label the edges of the island $I_{f}$ by the sequence of vertices and edges $\left\{\widetilde{v}_{1}, \widetilde{e}_{1}, \widetilde{v}_{2}, \widetilde{e}_{2}, \ldots, \widetilde{v}_{n}, \widetilde{e}_{n}\right\}$. Then the map $\varpi$ is defined on $I_{f}$ by sending the edges $\widetilde{e}_{i}$ to the edges $e_{i}$ and collapsing the edges $\widetilde{v}_{i}$ to the vertices $v_{i}$.

Bridges: Let $e$ be an edge in $K$; associate to it a rectangle $B_{e}=[0,1] \times[0,1]$ which we call a bridge in $\widetilde{K}$. If $e$ is contained in the boundaries of two $n$-gons $f$ and $f^{\prime}$, then $B_{e}$ will join $I_{f}$ and $I_{f^{\prime}}$ by attaching $\{0\} \times[0,1]$ to the edge of $I_{f}$ labelled ' $\tilde{e}$ ' and $\{1\} \times[0,1]$ to the edge of $I_{f^{\prime}}$ also labelled ' $\tilde{e}$ '. The map $\varpi$ collapses the bridge $B_{e}$ by sending $\{a\} \times[0,1]$ homeomorphically onto $e$ for every $a \in[0,1]$.

If $e$ is on the boundary of $K$, let $f$ be the single $n$-gon whose boundary contains $e$. We attach the bridge $B_{e}$ to $I_{f}$ by sending $\{0\} \times[0,1]$ to the edge of $I_{f}$ labelled ' $e$ '. Again $\varpi$ is defined on $B_{e}$ by sending $\{a\} \times[0,1]$ homeomorphically onto $e$. In either case, label the sides $[0,1] \times\{0\}$ and $[0,1] \times\{1\}$ by the vertices they map onto under $\varpi$.

Moats: For each vertex $v$ we construct an associated 2-cell $M_{v}$ which we call a moat. First, let $v$ be a vertex in the interior of $K$, let $\left\{f_{1}, \ldots, f_{m}\right\}$ be the circuit 


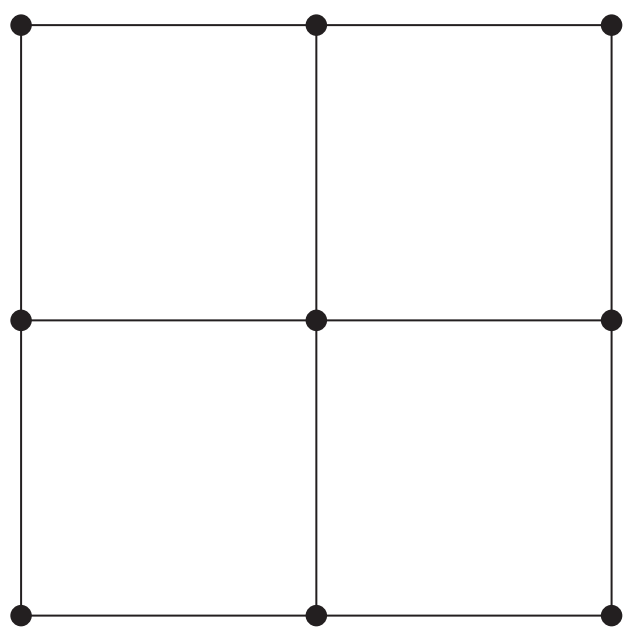

Figure 2. The image of a path, and a filling disk in $K$.

of faces surrounding $v$, and let $\left\{e_{1}, \ldots, e_{m}\right\}$ be the sequence of edges attached to $v$ where $f_{i} \cap f_{i+1} \supset e_{i}$. Then in the union of bridges and islands there is a circuit formed by the edges labelled $\widetilde{v}$ in the islands $I_{f_{i}}$ and the bridges $B_{e_{i}}$. For every such $v$ let $M_{v}$ be a $2 m$-gon filling the corresponding circuit.

Now let $v$ be a vertex on the boundary of $K$. Again, $v$ is surrounded by faces $\left\{f_{1}, \ldots, f_{m}\right\}$ and is attached to edges $\left\{e_{1}, \ldots, e_{m-1}\right\}$ where $f_{i} \cap f_{i+1} \supset e_{i}$. Let $e_{0}$ and $e_{m}$ be the edges on $\partial K$ which are attached to $v$. For each such vertex, add to the union of islands, bridges and moats formed so far, an edge connecting the terminal vertex $(1,1)$ of $B_{e_{0}}$ to the initial vertex $(1,0)$ of $B_{e_{m}}$. Finally, attach the moat $M_{v}($ a $(2 m+2)$-gon $)$ to the circuit formed by the edges labelled $\widetilde{v}$ in the islands $I_{f_{i}}$ and the bridges $B_{e_{i}}$, and this additional edge we have just attached.

The map $\varpi: \widetilde{K} \rightarrow K$ collapses the moat $M_{v}$ to the vertex $v$.

The union of these islands, bridges, and moats is our cellular 2-disk $\widetilde{K}$. Note that the boundary of $\widetilde{K}$ consists of edges labelled $\widetilde{v}_{i}$ and $\widetilde{e}_{i}$ where the $v_{i}$ 's and $e_{i}$ 's are the vertices and edges of $\partial K$. If it helps, consider a specific example, where $\pi(p)$ is the boundary of four cells as in Figure 2.

Each of the 2-cells will be covered by octagons in $\widetilde{K}$, the edges are covered by rectangles, and the vertices are covered by a variety of $n$-gons (see Figure 3 ).

We begin to define $\widetilde{\phi}: \widetilde{K} \rightarrow \mathcal{Y}$ by describing the image of the boundary. For each $i$, send the edge of $\partial \widetilde{K}$ which is labelled $\widetilde{e}_{i}$ to the edge in $p$ projecting to $e_{i}$ in $\pi(p)$. The edges of $\partial \widetilde{K}$ labelled by vertices $\widetilde{v}_{i}$ are then sent to the edge paths of $p$ contained in $\pi^{-1}(v)$. Thus we have a well-defined map $\widetilde{\phi}: \partial \widetilde{K} \rightarrow p$ and we need to extend this to a map $\widetilde{\phi}: \widetilde{K} \rightarrow \mathcal{Y}-C$.

For each island $I_{f} \subset \widetilde{K}$, define $\widetilde{\phi}\left(I_{f}\right)$ to be any 2-disk in $\pi^{-1}(\phi(f))$ whose closure does not intersect $D$. (This is always possible since $D$ is compact, and 


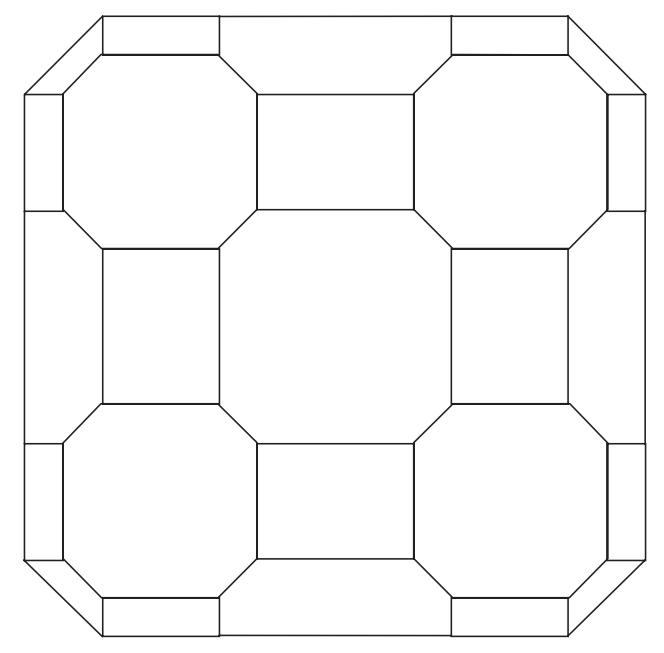

Figure 3. The corresponding collection $\widetilde{K}$.

$\pi^{-1}(\phi(f))$ is a countably infinite number of disjoint disks.)

Now consider a bridge $B_{e}$ in $\widetilde{K}$. The $\widetilde{\phi}$-image of two sides of this bridge are already determined; $\{0\} \times[0,1]$ and $\{1\} \times[0,1]$ are the two sides of the bridge that either connect two islands (if $e$ is an interior edge) or an island to the boundary of $\widetilde{K}$ (if $e$ is on the boundary of $K$ ). The barycenters of these two edges are mapped by $\widetilde{\phi}$ to vertices in the Cayley graph $\pi^{-1}\left(b_{\phi(e)}\right)$ which lie outside of $\widehat{D}_{\phi(e)}$. By our choice of $\widehat{D}_{\phi(e)}$ there is a path in $\pi^{-1}\left(b_{\phi(e)}\right)-\widehat{D}_{\phi(e)}$ connecting these two vertices. Send the bridge $B_{e}$ to the collection of 2 -cells induced by this path.

At this stage the $\widetilde{\phi}$-images of the boundaries of the moats have been determined by where $\phi$ sends the boundary of $\widetilde{K}$, the islands and the bridges. If $\varpi$ maps the moat $M_{v}$ to a vertex $v \in K$, then $\widetilde{\phi}\left(\partial M_{v}\right) \subset \pi^{-1}(\phi(v))-D_{\phi(v)}$. But the $\pi$-pre-images of vertices in $\mathcal{X}$ are 1 -connected at infinity, so one can extend the map $\left.\widetilde{\phi}\right|_{\partial M_{v}}$ to a map $\left.\widetilde{\phi}\right|_{M_{v}}$ where $\widetilde{\phi}\left(M_{v}\right) \subset \pi^{-1}(\phi(v))-C_{\phi(v)}$.

\section{Background on Artin groups}

Artin groups are a vexing collection of groups. Artin groups of finite type (those whose Coxeter quotients are finite) arise naturally in topology as fundamental groups of complex hyperplane complements and as fundamental groups of $(2, n)$ torus link complements. The Braid groups are Artin groups of finite type with Coxeter quotient the symmetric groups. While much is known about Artin groups of finite type, little is known about Artin groups in general. Charney and Davis have introduced a promising approach to the study of arbitrary Artin groups, viewing them as products - in a very vague sense outlined below — of Artin 
groups of finite type.

Let $A_{\mathcal{G}}$ be an Artin group with defining graph $\mathcal{G}$. In [10], Charney and Davis describe a complex $\Phi_{\mathcal{G}}$ on which $A_{\mathcal{G}}$ acts. Let $\mathcal{S}^{f}$ be the poset of complete subgraphs of $\mathcal{G}$ which correspond to Artin groups of finite type, ordered by inclusion. The empty subgraph is included in $\mathcal{S}^{f}$, hence the geometric realization of $\mathcal{S}^{f}$ is the cone over the barycentric subdivision of $\widehat{\mathcal{G}}$ where the cone point corresponds to $\emptyset$. Let

$$
\Phi_{\mathcal{G}}=\left\{a A_{\mathcal{C}} \mid a \in A_{\mathcal{G}}, \mathcal{C} \in \mathcal{S}^{f}\right\}
$$

be the geometric realization of the poset of indicated cosets, ordered by inclusion. Clearly $A_{\mathcal{G}}$ acts on $\Phi_{\mathcal{G}}$ on the left with a copy of $\mathcal{S}^{f}$ as fundamental domain. The stabilizers of the cells of $\Phi_{\mathcal{G}}$ under this action are conjugates of the Artin groups of finite type corresponding to $\left\{\mathcal{C} \in \mathcal{S}^{f}\right\}$.

Using work of Haefliger on complexes of groups, Charney and Davis establish that $\Phi_{\mathcal{G}}$ is 1-connected. The complex $\Phi_{\mathcal{G}}$ has a natural cubical structure, and so it inherits a piecewise Euclidean metric such that each cube isometric to the unit Euclidean cube of the appropriate dimension. If for each complete $\operatorname{subgraph} \mathcal{C} \subset \mathcal{G}$, the associated Artin group $A_{\mathcal{C}}$ is of finite type, then $A_{\mathcal{G}}$ is said to be of $F C$ type. The "FC" stands for "flag complex"; the geometric realization of $\mathcal{S}$ " is a flag complex if and only if $A_{\mathcal{G}}$ is of FC type. In Theorem 4.3.5 of [10], Charney and Davis show that $\Phi_{\mathcal{G}}$ with its piecewise cubical metric is $\operatorname{CAT}(0)$ if and only if $A_{\mathcal{G}}$ is of FC type. In this case it follows that $\Phi_{\mathcal{G}}$ is contractible; Charney and Davis conjecture that $\Phi_{\mathcal{G}}$ is always contractible (Conjecture 2 in [10]).

Our arguments exploit the fact that the cell stabilizers for the action of $A_{\mathcal{G}}$ on $\Phi_{\mathcal{G}}$ are Artin groups of finite type. The connectivity at infinity properties of finite type Artin groups are understood by work of Squier and Bestvina (see [25] and [1]). Let $|\mathcal{G}|$ be the rank of $\mathcal{G}$, that is, the number of vertices in $\mathcal{G}$.

Theorem 5.1. Let $A_{\mathcal{G}}$ be an Artin group of finite type. Then $A_{\mathcal{G}}$ is a duality group of dimension $|\mathcal{G}|$ and is $(|\mathcal{G}|-2)$-connected at infinity.

Squier's (little known) work in [25] only establishes duality, hence only homological connectivity at infinity; the homotopy at infinity result is done by Bestvina [25] using an insightful geometric argument.

One might hope that Theorem A could be applied to the action of $A_{\mathcal{G}}$ on $\Phi_{\mathcal{G}}$ to establish connectivity at infinity properties for Artin groups. Regrettably this is not the case. The essential difficulty is that the stabilizers are not well behaved. For example, the isotropy groups for the cone points in $\Phi_{\mathcal{G}}$ are trivial, not infinite; the isotropy groups of the other vertices of $\Phi_{\mathcal{G}}$ are Artin groups of dimensions $1,2,3, \ldots$ up to $\operatorname{dim}\left(\mathcal{S}^{f}\right)$. Because the vertex stabilizers are not always top dimensional, nor are edge stabilizers of codimension one, etc., the spectral sequence used in $\S 3$ doesn't have zeros below the critical diagonal. To avoid this difficulty, we use a different $A_{\mathcal{G}}$-equivariant filtration of $\Phi_{\mathcal{G}}$ other than by skeleta, and this filtration induces a more tractable spectral sequence. 
Let $\operatorname{dim}\left(\Phi_{\mathcal{G}}\right)=n$. We reserve the letter $\mathcal{C}$ for subgraphs in $\mathcal{S}^{f}$; so $A_{\mathcal{C}}$ will always denote an Artin group of finite type contained in $A_{\mathcal{G}}$. Let $\Phi_{\mathcal{G}}^{0}$ be the geometric realization of the poset of $\operatorname{cosets}\left\{a A_{\mathcal{C}}|| \mathcal{C} \mid=n\right\}$; this is a discrete collection of points that will fill the role of a 0 -skeleton. In general, $\Phi_{\mathcal{G}}^{i}$ is the geometric realization of the poset $\left\{a A_{\mathcal{C}}|| \mathcal{C} \mid \geq n-i\right\}$. At the final stage we add the cone points forming $\Phi_{\mathcal{G}}^{n}=\Phi_{\mathcal{G}}$. Equivalently one can think of the complex $\Phi_{\mathcal{G}}^{i}$ as the union of the fixed point sets of the conjugates of $\left\{A_{\mathcal{C}}|| \mathcal{C} \mid \geq n-i\right\}$. From this point of view one can talk about the rank of a cell as being the rank of the isotropy group of the cell, and one sees that our filtration is given by "corank."

Following Charney and Davis, we let $\mathcal{S}_{\geq \mathcal{C}}^{f}$ denote the subposet $\left\{\mathcal{C}^{\prime} \in \mathcal{S}^{f} \mid \mathcal{C}^{\prime} \supseteq\right.$ $\mathcal{C}\} \subset \mathcal{S}^{f}$. The fundamental domain for the action of $A_{\mathcal{G}}$ on $\Phi_{\mathcal{G}}^{i}$ is $\bigcup_{|\mathcal{C}|=n-i} \mathcal{S}_{\geq \mathcal{C}}^{f}$. We let $\mathcal{S}_{>\mathcal{C}}^{f}$ be the subposet $\left\{\mathcal{C}^{\prime} \in \mathcal{S}^{f} \mid \mathcal{C}^{\prime} \supset \mathcal{C}\right\} \subset \mathcal{S}^{f}$.

\section{Simple connectivity at infinity for Artin groups}

In this section we outline how the techniques of $\S 4$ can be modified to prove Theorem B.

Theorem B. Let $A_{\mathcal{G}}$ be an Artin group, let $\widehat{\mathcal{G}}$ be the complex described above, and assume that $\mathcal{G}$ is not a single vertex or edge. If $\widehat{\mathcal{G}}$ is 1 -connected and contains no cut vertex, then $A_{\mathcal{G}}$ is 1-connected at infinity.

Our argument will use the action of $A_{\mathcal{G}}$ on a subcomplex of its modified Deligne complex. Assuming we are in the situation described in Theorem B, the link of any cone point in $\Phi_{\mathcal{G}}$ is simply-connected. Hence removing all the cone points from $\Phi_{\mathcal{G}}$ leaves a simply-connected space. Similarly the link of any vertex of rank 4 or higher is simply-connected (see Lemma 4.3 .1 in [10]), so they may also be removed. The resulting space deformation retracts onto the simply-connected subcomplex of $\Phi_{\mathcal{G}}$ consisting of cells of rank 1,2 and 3. We denote this complex $\bar{\Phi}_{\mathcal{G}}$.

Let $\mathcal{Y}_{\mathcal{G}}$ be the space covering $\bar{\Phi}_{\mathcal{G}}$ on which $A_{\mathcal{G}}$ acts freely; as before let $\pi: \mathcal{Y}_{\mathcal{G}} \rightarrow \bar{\Phi}_{\mathcal{G}}$ be the $A_{\mathcal{G}}$-equivariant cellular projection. In our proof of Theorem B we use the following fact from [7].

Lemma 6.1. Given the conditions of Theorem B, Lk $(v, \widehat{\mathcal{G}})$ is connected for any vertex $v \in \widehat{\mathcal{G}}$, and $L k(e, \widehat{\mathcal{G}})$ is non-empty for any edge $e \in \widehat{\mathcal{G}}$.

Given a compact set $C \subset \mathcal{Y}_{\mathcal{G}}$, we find the 1-connectivity subcomplex $D \supset C$ as in $\S 4$ by adding appropriate subcomplexes in the pre-images of cells in $\bar{\Phi}_{\mathcal{G}}$.

Any edge in $\bar{\Phi}_{\mathcal{G}}$ connects vertices of different ranks. An edge path in $\bar{\Phi}_{\mathcal{G}}$ is standard if it never passes through a vertex of rank 1. Standard edge paths 
most closely match the intuition of previous sections as they never contain an edge whose stabilizer is not one ended, and every other vertex of a standard edge path has rank 3. Thus, in a standard edge path, every other vertex is stabilized by a group which is simply connected at infinity.

Lemma 6.2. Let $C$ be a compact set in $\mathcal{Y}_{\mathcal{G}}, D$ the associated compact set containing $C$, and let $p$ be an edge path in $\mathcal{Y}_{\mathcal{G}}-D$. Then $p$ can be homotoped in $\mathcal{Y}_{\mathcal{G}}-C$ to an edge path $p^{\prime}$ where $\pi\left(p^{\prime}\right)$ is a standard edge path in $\bar{\Phi}_{\mathcal{G}}$.

Proof. If $\pi(p)$ passes through a rank 1 vertex $v$, it must arrive and depart through vertices of higher rank. Because $v$ is a rank 1 vertex, its stabilizer is $\mathbb{Z}$, generated by a conjugate of the generator corresponding to some $\nu \in \widehat{\mathcal{G}}$. The portion of the link of $\pi(v) \subset \Phi_{\mathcal{G}}$ consisting of cells of rank $>1$ is combinatorially isomorphic to $\operatorname{Lk}(\nu, \widehat{\mathcal{G}})=\mathcal{S}_{>\nu}^{f}$. By Lemma 6.1, this is connected, so the portion of the link of $\pi(v)$ consisting of cells of rank $>1$ is also connected. Because quotient maps are continuous, $\pi^{-1}\left(\operatorname{Lk}\left(v, \bar{\Phi}_{\mathcal{G}}\right)\right)$ is also connected. So we can homotope $p$ to a path which avoids $v$ and only passes through vertices of higher rank.

Proof of Theorem B. By Lemma 6.2 we may assume that the path $\pi(p) \subset \bar{\Phi}_{\mathcal{G}}$ is a standard edge path. We use essentially the same argument as in Lemma 4.1 to reduce ourselves to the case where $\pi(p)$ is a simple edge path circuit in $\bar{\Phi}_{\mathcal{G}}$. The case where $\pi(p)$ is a tree is the only sticky situation. If a valence 1 vertex $v \in \pi(p)$ is of rank 2 , then $\pi^{-1}(v)$ is 0 -connected at infinity, but not 1-connected at infinity. This is a problem since in the proof of Lemma 4.1 we created a loop in $\pi^{-1}(v)-D$ which we filled in $\pi^{-1}(v)-C$. (This situation is illustrated in Figure 1.) However, a rank 2 vertex in $\bar{\Phi}_{\mathcal{G}}$ corresponds to the barycenter of an edge $e$ in $\widehat{\mathcal{G}}$. By Lemma $6.1, e$ is contained in the boundary of some 2 -simplex $\sigma \subset \widehat{\mathcal{G}}$. Let $w$ be a vertex adjacent to $v$ in $\Phi_{\mathcal{G}}$ corresponding to the barycenter of $\sigma$. Because $\pi^{-1}(v)$ injects into $\pi^{-1}(w)$, which is 1-connected at infinity, the loop we create in $\pi^{-1}(v)$ can be freely homotoped into $\pi^{-1}(w)$ where it can be filled outside of $C$.

We may now assume that $\pi(p)$ is a standard, simple edge path circuit, and because $\bar{\Phi}_{\mathcal{G}}$ is simply connected, $\pi(p)$ can be filled in $\bar{\Phi}_{\mathcal{G}}$. That is to say, there is a combinatorial disk $K$ and a combinatorial map $\phi:(K, \partial K) \rightarrow\left(\bar{\Phi}_{\mathcal{G}}, \pi(p)\right)$.

At this stage we apply the techniques from $\S 4$ to the current situation. That is, we construct $\widetilde{K}$ by blowing up the cells of $K$, and then construct a map $\varpi:(\widetilde{K}, \partial \widetilde{K}) \rightarrow\left(\mathcal{Y}_{\mathcal{G}}, p\right)$. We start by identifying the "islands"; recall that in the original argument islands were 2-cells stabilized by -1-connected at infinity groups. We say the rank of any vertex $v \in K$ is the rank of its $\phi$-image in $\bar{\Phi}_{\mathcal{G}}$. Every 2-cell in $\bar{\Phi}_{\mathcal{G}}$ contains a vertex $v$ of rank 1 ; thus there is a copy of $\mathbb{Z}$ stabilizing the closed star of $v$ in $\bar{\Phi}_{\mathcal{G}}$. The role of islands is played by the stars of the rank 1 vertices in $K$. These stars are combinatorial disks, and their images in $\bar{\Phi}_{\mathcal{G}}$ are stabilized by copies of $\mathbb{Z}$. Thus to construct $\widetilde{K}$ and a map $\widetilde{\phi}:(\widetilde{K}, \partial \widetilde{K}) \rightarrow\left(\mathcal{Y}_{\mathcal{G}}, p\right)$ we begin by choosing one of the countably many disks in $\pi^{-1}(\phi(\operatorname{St}(v)))$ which misses $D$. 
Next we join the islands by bridges, where the brides correspond to edge pairs with the three vertices of ranks 3,2 and 3 respectively. The isotropy group of the edge pair is a finite type Artin group of rank 2, hence it's one ended. This allows us to connect the lifts of adjoining islands by paths outside of $D$.

Finally, the moats correspond to rank 3 vertices in $K$, whose images in $\bar{\Phi}_{\mathcal{G}}$ are stabilized by Artin groups that are simply connected at infinity. The moats fill edge path circuits formed in $\widetilde{K}$ by the bridges and islands; because the vertex groups involved are simply connected at infinity, we can map these moats to embedded disks in simply connected at infinity subcomplexes of $\mathcal{Y}_{\mathcal{G}}$.

This same technique can be applied to graph products of groups; we quickly outline the approach to this situation. Let $\mathcal{G}$ be a finite simplicial graph with groups $G_{v}$ associated to the vertices $v \in \mathcal{G}^{(0)}$. The graph product $G_{\mathcal{G}}$ is the free product of the $G_{v}$ 's modulo relations implying that adjacent vertex groups commute. In this situation, we let $\widehat{\mathcal{G}}$ be the flag complex induced by $\mathcal{G}$.

Theorem 6.3. Let $G_{\mathcal{G}}$ be a graph product of finitely presented infinite groups. If $\widehat{\mathcal{G}}$ is 1-connected, and if $G_{v}$ is 1 -ended for any cut vertex $v \in \widehat{\mathcal{G}}$, then $G_{\mathcal{G}}$ is 1-connected at infinity.

The argument begins by noting that the direct sum of three infinite, finitely presented groups is simply connected at infinity [18]. Next one constructs an (improper) action of the graph product on a cell complex, similar to the action of an Artin group on its modified Deligne complex; this construction is a minor modification of the techniques of Moussong-Charney-Davis and is written down in [21]. If $\widehat{\mathcal{G}}$ has no cut vertex, one then proceeds as in the argument above. If $\widehat{\mathcal{G}}$ has a cut vertex $v$, then $\widehat{\mathcal{G}}$ can be expressed as the union of simply connected components which share the common vertex $v$. An inductive argument shows that $G_{\mathcal{G}}$ decomposes as the fundamental group of a graph of groups. The vertex groups correspond to subgroups associated with subgraphs $\mathcal{G}^{\prime} \subset \mathcal{G}$ where $\mathcal{G}^{\prime}$ does not contain cut vertices and $\widehat{\mathcal{G}}^{\prime}$ is simply connected. The edge groups in the graph of groups decomposition consist of the $G_{v}$ 's where $v$ is a cut vertex. (See the argument in $\S 5$ in [22].) The argument above establishes that each vertex group in the arboreal decomposition is simply connected at infinity; because the edge groups in this decomposition correspond to one-ended groups, $G_{\mathcal{G}}$ is simply connected at infinity by Theorem A. 


\section{Which Artin groups are duality groups?}

Here we prove

Theorem C. Let $A_{\mathcal{G}}$ be an Artin group whose modified Deligne complex $\Phi_{\mathcal{G}}$ is contractible. If $\widehat{\mathcal{G}}$ is an $(n-1)$-dimensional Cohen-Macaulay complex, then $A_{\mathcal{G}}$ is a duality group of dimension $n$ with $\mathbb{Z}$-free dualizing module.

Recall that an $n$-dimensional simplicial complex $\widehat{\mathcal{G}}$ is Cohen-Macaulay if its reduced homology is concentrated in dimension $n$, and for each simplex $\sigma \subset \widehat{\mathcal{G}}$, the reduced homology of $\operatorname{Lk}(\sigma, \widehat{\mathcal{G}})$ is concentrated in dimension $n-|\sigma|-1$.

The fact that the dualizing module is $\mathbb{Z}$-free (and not just $\mathbb{Z}$-torsion free) will follow from the fact that the dualizing module for Artin groups of finite type is $\mathbb{Z}$-free. (See Squier's comments in the paragraph following Lemma 8.4 of [25].)

Proof. Because Artin groups of finite type are $F P$, and $\Phi_{\mathcal{G}}$ is assumed to be contractible, $A_{\mathcal{G}}$ is $F P$. Thus it suffices to establish that the cohomology of $A_{\mathcal{G}}$ with $\mathbb{Z} A_{\mathcal{G}}$ coefficients is concentrated in top dimension and is $\mathbb{Z}$-free.

We express $H^{*}\left(A_{\mathcal{G}}, \mathbb{Z} A_{\mathcal{G}}\right)$ in terms of the equivariant cohomology for the action of $A_{\mathcal{G}}$ on $\Phi_{\mathcal{G}}, H_{A_{\mathcal{G}}}^{*}\left(\Phi_{\mathcal{G}}\right)$. Recall that our filtration of $\Phi_{\mathcal{G}}$ is by $A_{\mathcal{G}}$-equivariant subcomplexes, but not by skeleta, so we need to analyze the spectral sequence induced by this filtration.

The relative chains $C\left(\Phi_{\mathcal{G}}^{p}, \Phi_{\mathcal{G}}^{p-1}\right)$ can be expressed in terms of induced modules based at the fundamental domain: $\bigoplus_{|\mathcal{C}|=n-p} C\left(\mathcal{S}_{\geq \mathcal{C}}^{f}, \mathcal{S}_{>\mathcal{C}}^{f}\right) \uparrow_{A_{\mathcal{C}}}^{A_{\mathcal{G}}}$.

Let $F$ be a finite free resolution of $\mathbb{Z}$ as a $\mathbb{Z} A_{\mathcal{G}}$-module. Then

$$
\begin{gathered}
\operatorname{Hom}_{A_{\mathcal{G}}}\left(F, \operatorname{Hom}_{\mathbb{Z}}\left(C\left(\Phi_{\mathcal{G}}^{p}, \Phi_{\mathcal{G}}^{p-1}\right), \mathbb{Z} A_{\mathcal{G}}\right)\right) \\
=\bigoplus_{|\mathcal{C}|=n-p} \operatorname{Hom}_{A_{\mathcal{G}}}\left(F, \operatorname{Hom}_{\mathbb{Z}}\left(C\left(\mathcal{S}_{\geq \mathcal{C}}^{f}, \mathcal{S}_{>\mathcal{C}}^{f}\right) \uparrow_{\left.\left.\uparrow_{\mathcal{C}}^{A_{\mathcal{G}}}, \mathbb{Z} A_{\mathcal{G}}\right)\right)}\right.\right. \\
=\bigoplus_{|\mathcal{C}|=n-p} \operatorname{Hom}_{A_{\mathcal{C}}}\left(F, \operatorname{Hom}_{\mathbb{Z}}\left(C\left(\mathcal{S}_{\geq \mathcal{C}}^{f}, \mathcal{S}_{>\mathcal{C}}^{f}\right), \mathbb{Z} A_{\mathcal{G}}\right)\right)
\end{gathered}
$$

It follows that

$$
E_{1}^{p q}=H_{A_{\mathcal{G}}}^{p+q}\left(\Phi_{\mathcal{G}}^{p}, \Phi_{\mathcal{G}}^{p-1}\right)=\bigoplus_{|\mathcal{C}|=n-p} H_{A_{\mathcal{C}}}^{p+q}\left(\mathcal{S}_{\geq \mathcal{C}}^{f}, \mathcal{S}_{>\mathcal{C}}^{f}\right)=\bigoplus_{|\mathcal{C}|=n-p} H^{q}\left(A_{\mathcal{C}}, H^{p}\left(\mathcal{S}_{\geq \mathcal{C}}^{f}, \mathcal{S}_{>\mathcal{C}}^{f}\right)\right)
$$

where we've suppressed the $\mathbb{Z} A_{\mathcal{G}}$-coefficients to avoid notational clutter. The pair $\left(\mathcal{S}_{\geq \mathcal{C}}^{f}, \mathcal{S}_{>\mathcal{C}}^{f}\right)$ is a cone over the link of the simplex described by $\mathcal{C}$ in the defining complex $\widehat{\mathcal{G}}$. Because $\widehat{\mathcal{G}}$ is Cohen-Macaulay, $\left(\mathcal{S}_{\geq \mathcal{C}}^{f}, \mathcal{S}_{>\mathcal{C}}^{f}\right)$ has the homology of a wedge 
of spheres of dimension $p$, so the relative homology is trivial except in dimension $p$ where it's free abelian. Thus

$$
H^{p}\left(\left(\mathcal{S}_{\geq \mathcal{C}}^{f}, \mathcal{S}_{>\mathcal{C}}^{f}\right), \mathbb{Z} A_{\mathcal{G}}\right)=\operatorname{Hom}\left(H_{p}\left(\mathcal{S}_{\geq \mathcal{C}}^{f}, \mathcal{S}_{>\mathcal{C}}^{f}\right), \mathbb{Z} A_{\mathcal{G}}\right)=\bigoplus \mathbb{Z} A_{\mathcal{G}}
$$

So

$$
E_{1}^{p q}= \begin{cases}\mathbb{Z} \text {-free } & q=n-p \\ 0 & \text { otherwise }\end{cases}
$$

and therefore the entire spectral sequence lies in total degree $p+q=n$. Because all the entries below the $n^{\text {th }}$-diagonal are zero, $H^{q}\left(A_{\mathcal{G}}, \mathbb{Z} A_{\mathcal{G}}\right)=0$ up to dimension $n$; since each $A_{\mathcal{C}}$ is an $F P$ group, cohomology commutes with direct sums, hence $H^{n}\left(A_{\mathcal{G}}, \mathbb{Z} A_{\mathcal{G}}\right)$ is $\mathbb{Z}$-free.

Corollary 7.1. If $A_{\mathcal{G}}$ is an Artin group of FC-type, and $\widehat{\mathcal{G}}$ is Cohen-Macaulay, then $A_{\mathcal{G}}$ is a duality group.

We note that the spectral sequence constructed in the proof of Theorem C works with any coefficients. In particular, in combination with Squier's results on the integral cohomology of Artin groups of finite type [25], it might prove useful for computing the integral cohomology of Artin groups of infinite type. We also mention that our proof establishes that if $\Phi_{\mathcal{G}}$ is contractible, and if the $n$-skeleton of $\widehat{\mathcal{G}}$ is Cohen-Macaulay, then $A_{\mathcal{G}}$ is $(n-1)$-acyclic at infinity (even if $A_{\mathcal{G}}$ might not be a duality group).

\section{Acknowledgment}

We thank Mike Davis for helpful conversations on this material during a visit to Ohio State.

\section{References}

[1] M. Bestvina, Nonpositively curved aspects of Artin groups of finite type, Geometry and Topology 3 (1999), 269-302.

[2] M. Bestvina and N. Brady, Morse theory and finiteness properties of groups, Invent. Math. 129 (1997), 445-470.

[3] M. Bestvina and M. Feighn, The topology at infinity of $\operatorname{Out}\left(F_{n}\right)$, preprint.

[4] R. Bieri, Homological dimension of discrete groups, Queen Mary College Mathematics Notes, London 1976.

[5] R. Bieri and B. Eckmann, Groups with homological duality generalizing Poincaré duality, Invent. Math 20 (1973), 103-124.

[6] A. Borel and J-P. Serre, Corners and arithmetic groups, Comment. Math. Helv. 48 (1974), $74-83$.

[7] N. Brady and J. Meier, Connectivity at infinity for right angled Artin groups, to appear, Trans. Amer. Math. Soc. 
[8] K.S. Brown, Cohomology of Groups, Springer-Verlag, 1982.

[9] K.S. Brown, Finiteness properties of groups, J. Pure Appl. Alg. 44 (1987), 45-75.

[10] R. Charney and M.W. Davis, The $K(\pi, 1)$-problem for hyperplane complements associated to infinite reflection groups, Jour. Amer. Math. Soc. 8 (1995), 597-627.

[11] J.M. Corson, Complexes of groups, Proc. Lond. Math. Soc. (3) 65 (1992), 199-224.

[12] M.W. Davis, The cohomology of a Coxeter group with group ring coefficients, Duke Math. J. 91 (1998), 297-314. [Correction: Duke Math. J. 95 (1998), 635].

[13] M.W. Davis, Poincaré Duality Groups, to appear in Surveys in Surgery Theory.

[14] P. Deligne, Les immeubles des groupes de tresses généralisés, Invent. Math. 17 (1972), 273-302.

[15] R. Geoghegan, Topological Methods in Group Theory, book in progress.

[16] A. Haefliger, Extensions of complexes of groups, Ann. Inst. Fourier 42 (1992), 275-311.

[17] C. Hog-Angeloni, W. Metzler and A.J. Sieradski, Two-dimensional Homotopy and Combinatorial Group Theory, LMS Lect. Notes 197, Cambridge University Press, 1993.

[18] B. Jackson, End invariants of group extensions, Topology 21 (1982), 71-81.

[19] B. Jackson, End invariants of amalgamated free products, J. Pure Appl. Alg. 23 (1982), 233-242.

[20] S. Mardešić and J. Segal, Shape Theory, North-Holland, 1982.

[21] J. Meier, When is the graph product of hyperbolic groups hyperbolic? Geom. Dedicata 61 (1996), 29-41.

[22] J. Meier, H. Meinert and L. VanWyk, The $\Sigma^{2}$-invariants for graph products of indicable groups, Top. Appl. 99 (1999), 41-65.

[23] H. Meinert, Actions on 2-complexes and the homotopical invariant $\Sigma^{2}$ of a group, J. Pure Appl. Alg. 119 (1997), 297-317.

[24] G.P. Scott and C.T.C. Wall, Topological methods in group theory, Homological group theory, LMS Lect. Notes 36, Cambridge University Press (1979) 137-203.

[25] C. Squier, The homological algebra of Artin groups, Math. Scand. 75 (1995), 5-43.

Kenneth S. Brown

Department of Mathematics

Cornell University

Ithaca NY 14853

USA

e-mail: kbrown@math.cornell.edu
John Meier

Department of Mathematics

Lafayette College

Easton PA 18042

USA

e-mail: meierj@lafayette.edu

(Received: October 30, 1998) 